\title{
MODERATE DEVIATION AND EXIT TIME ESTIMATES FOR STATIONARY LAST PASSAGE PERCOLATION
}

\author{
MANAN BHATIA
}

\begin{abstract}
We consider planar stationary exponential Last Passage Percolation in the positive quadrant with boundary weights. For $\rho \in(0,1)$ and points $v_{N}=\left((1-\rho)^{2} N, \rho^{2} N\right)$ going to infinity along the characteristic direction, we establish right tail estimates with the optimal exponent for the exit time of the geodesic, along with optimal exponent estimates for the upper tail moderate deviations for the passage time. For the case $\rho=\frac{1}{2}$ in the stationary model, we establish the lower bound estimate with the optimal exponent for the lower tail of the passage time. Our arguments are based on moderate deviation estimates for point-to-point and point-to-line exponential Last Passage Percolation which are obtained via random matrix estimates.
\end{abstract}

\section{INTRODUCTION}

The planar exponential Last Passage Percolation (LPP) model is an important and canonical integrable model in the (1+1)-dimensional KPZ universality class. The model has been mainly studied for three initial conditions- step, flat and stationary. Currently, there are two main approaches for analysing these models- the first relying on using the random matrix connections for the models with the step and flat initial conditions to obtain concentration for the passage time $[9,5,6,8]$. The second approach relies on using duality along with the Burke property for the stationary initial condition [3, 15, 18].

For the stationary initial condition, the exit time is an important quantity which has been used to establish the correct order of the variance of the stationary passage time along the characteristic line [3], along with optimal estimates for the coalescence time of two semi-infinite geodesics in exponential LPP [15, 18]. Until very recently, only suboptimal tail estimates were available for the exit time [3]. Some estimates for the exit time have also been obtained using Fredholm determinantal formulae [11, 12], and the lower bound with the optimal exponent for the exit time is known [17, 4]. Though there are exact correspondences to the eigenvalues of certain random matrices for the passage time in the case of the step and flat initial conditions $[13,5,14]$, no such correspondence is known in the case of the stationary initial condition which makes it difficult to directly use inputs from the random matrix literature for its analysis.

In this paper, in Theorem 2.5 and Theorem 2.6, we show that one can use the known concentration estimates for point-to-point and point-to-line LPP originating from the random matrix connections [14, 9, 5] to obtain optimal moderate deviation concentration estimates for upper and lower tails of the passage time in the stationary model. In Theorem 2.4, we obtain optimal exponent right tail estimates for the exit time by using similar techniques. As a matter of fact, a possible proof of the optimal exponents via the transversal fluctuation estimates proved in [8] using the moderate deviation estimates coming from the random matrix connections, together with duality and Busemann functions was indicated in [18]; we, however, provide a direct proof using the moderate deviation estimates without appealing to duality and transversal fluctuation estimates.

As we were finishing up this paper, the paper [10] was posted on the arXiv where the optimal exponent right tail estimates for the exit time, as well as the optimal exponent upper tail estimates for the passage time for the stationary initial condition are obtained. The proof however, is different from ours- the approach is based on obtaining an exact formula for the moment generating function for the stationary passage time and then using it to obtain the other results. In contrast, our approach proceeds by using the random matrix estimates for the point-to-point and the point-to-line passage times and then using it to obtain the results for the stationary model. In a broader context, the two approaches mentioned at the beginning of the introduction have sometimes led to parallel results ([8], [15, 18] and [7], [2]), and this paper is also in the same spirit. 
The estimates for the lower bound on the upper tail and the upper bound on the lower tail of the stationary passage time are immediate from comparison with the point-to-point model. For the special case of $\rho=\frac{1}{2}$ in the stationary model, we were also able to obtain the estimate with the optimal exponent for the lower bound on the lower tail which is not available in the literature so far. As the reader will see, our proofs only depend on the moderate deviation estimates from random matrix connections together with the strict concavity of the shape function $(\sqrt{x}+\sqrt{y})^{2}$, and the same proofs are expected to work for other models where such estimates are known, e.g. stationary versions of Poissonian and Geometric LPP.

Outline of the paper. In Section 2, we give the precise definitions of the models that we are working with and provide the statements of our main results. In Section 3, we state known results relating to deviation estimates for the point-to-point and point-to-line passage times; we will be using these in our arguments. In Section 4, we prove Theorem 2.4, the tight upper bound on the upper tail of the stationary LPP exit time. The upper and lower tail estimates for the stationary last passage time- Theorem 2.5 and Theorem 2.6 are proved in Sections 5 and 6 respectively.

Acknowledgements. The author thanks Riddhipratim Basu for useful discussions and valuable encouragements. The author was supported by the KVPY fellowship from the Government of India, along with the LTVSP program at ICTS, Bangalore.

\section{Model Definitions AND main Results}

Definition 2.1 (Planar exponential LPP). Define a random field

$$
\omega=\left\{\omega_{v}: v \in \mathbb{Z}^{2}\right\} \text {. }
$$

where the $\omega_{v}$ are i.i.d. $\exp (1)$ random variables. Given $u, v \in \mathbb{Z}^{2}$ with $u \leq v$ (that is, $u$ is coordinate-wise smaller than $v$ ), for any up-right path $\gamma$ from $u$ to $v$, define the weight of the path $l(\gamma)$ as

$$
l(\gamma)=\sum_{w \in \gamma} \omega_{w} .
$$

For any $u \leq v$, define the point-to-point passage time $G(u, v)$ by the maximum of $l(\gamma)$ over all up-right paths from $u$ to $v$, and define it to be $-\infty$ otherwise. Call the a.s. unique path attaining the maximum as the geodesic from $u$ to $v$. In a similar manner, we can also define point-to-line passage times. To simplify notation later, we define the point-to-line passage time from a point to the line $\{x+y=0\}$ with a weight function. Namely, if $T$ is a possibly random weight function on the line $\{x+y=0\}$, and $v$ is a point above the line, then one can define the point-to-line passage time with initial condition $T$ as

$$
G_{T}(v)=\max _{u \in\{x+y=0\}}\{T(u)+G(u, v)\} .
$$

Note that if $T$ is identically 0 , then we denote the corresponding point-to-line passage time by $G_{0}(\cdot)$. Also note that we will be using the notation $\mathbb{P}(\cdot)$ when denoting probabilities for this model.

Note that though we defined the point-to-point and point-to-line passage times for the environment in Definition 2.1, these quantities can be similarly defined for other environments too. We now come to the stationary LPP model. The Totally Asymmetric Simple Exclusion Process (TASEP) started from a given initial configuration of particles and holes can be realized as a corresponding LPP model, and the stationary LPP model is the one corresponding to the TASEP started from a stationary distribution. Note that the TASEP and hence the stationary LPP model has a one parameter family of invariant measures parametrised by the particle density $\rho \in[0,1]$. We will be using two different representations of the stationary model. For clarity, we define them separately. Note that we use $\mathbf{0}$ for $(0,0)$.

Definition 2.2 (Boundary representation of stationary LPP with density $\rho \in(0,1)$ ). This model was introduced in [3] and is defined on the positive quadrant $\mathbb{Z}_{\geq 0}^{2}$. Let $\mathrm{e}_{1}, \mathrm{e}_{2}$ denote the unit vectors along the $x$ and $y$ axes respectively. Define a random field

$$
\omega^{\prime}=\left\{\omega_{v}^{\prime}: v \in \mathbb{Z}_{\geq 0}^{2}\right\}
$$

where the $\omega_{v}^{\prime}$ are independent random variables such that $\omega_{0}^{\prime}=0$ and $\omega_{v}^{\prime} \sim \exp (1)$ for all $v \in \mathbb{Z}_{>0}^{2}$. For the coordinate axes, we have that $\omega_{i_{1}}^{\prime} \sim \exp (1-\rho)$ and $\omega_{j \mathrm{e}_{2}}^{\prime} \sim \exp (\rho)$ for all $i, j \geq 1$. For any $v \in \mathbb{Z}_{\geq 0}^{2}$, define the stationary last passage time time $G_{\text {stat }}^{1}(v)$ as the maximum of $l(\gamma)$ over all up-right paths from $\mathbf{0}$ 
to $v$. Here, $l(\gamma)$ is defined as earlier by using the weights from the environment $\omega^{\prime}$. Call the a.s. unique path attaining the maximum as the stationary geodesic to $v$. When denoting probabilities for this model, we will use the notation $\mathbb{P}^{\rho}(\cdot)$.

Definition 2.3 (Point-to-line representation of stationary LPP with density $\rho \in(0,1)$ ). We take this model from [11]. The model is defined on the upper-right half-plane $\{x+y \geq 0\} \subseteq \mathbb{Z}^{2}$. Define the random field

$$
\omega^{\prime \prime}=\left\{\omega_{v}^{\prime \prime}: v \in \mathbb{Z}^{2}\right\},
$$

where the $\omega_{v}^{\prime \prime}$ are independent random variables such that $\omega_{v}^{\prime \prime} \sim \exp (1)$ for all $v \in\{x+y>0\}$, and $\omega_{v}^{\prime \prime}=0$ otherwise. For $v \in\{x+y=0\}$, let $\tau_{v}, \psi_{v}$ be random variables independent of each other and $\omega^{\prime \prime}$ with the marginals $\tau_{v} \sim \exp (1-\rho)$ and $\psi_{v} \sim \exp (\rho)$. We now define a weight function on the line $\{x+y=0\}$. Given $v \in\{x+y=0\}$ with $v=(t,-t)$, define

$$
T(v)= \begin{cases}0, & \text { for } t=0 \\ \sum_{s=1}^{t}\left(\tau_{(s,-s)}-\psi_{(s,-s)}\right), & \text { for } t>0 \\ -\sum_{s=t}^{-1}\left(\tau_{(s,-s)}-\psi_{(s,-s)}\right), & \text { for } t<0\end{cases}
$$

For $v \in\{x+y \geq 0\}$, define the the last passage time $G_{\text {stat }}^{2}(v)$ by

$$
G_{\text {stat }}^{2}(v)=G_{T}(v)=\max _{u \in\{x+y=0\}}\{T(u)+G(u, v)\}
$$

where the point-to-point passage times $G$ are computed using $\omega^{\prime \prime}$. Call the a.s. unique path attaining the maximum (starting from the line $\{x+y=0\}$ and ending at $v_{N}$ ) as the stationary geodesic to $v$. When denoting probabilities for this model, we will use the notation $\overline{\mathbb{P}}^{\rho}(\cdot)$.

The two representations are known to be equivalent, and a proof is provided in the appendix. We will mostly be working with the boundary representation of the stationary model, but the point-to-line representation will be used in the proof of Theorem 2.6 to obtain the lower bound for the lower tail for the stationary passage time for $\rho=\frac{1}{2}$.

Notational comments. We try to use the letters $C, c$ for constants in all the Lemmas, Propositions and Theorems to prevent cluttering due to an overuse of subscripts; we do not mean that all of the results are true with the same constants. Regarding boldface letters, if $x>0$, then $\mathbf{x}$ denotes $(x, 0)$ and $\mathbf{x}^{\uparrow}$ denotes $(x, 1)$. If $x<0$ then $\mathbf{x}$ denote $(0,-x)$ and $\mathbf{x}^{\uparrow}$ denotes $(1,-x)$. Finally, $\mathbf{0}$ denotes $(0,0)$. For a real variable $t \neq 0$, we use the notation $\mathfrak{t}$ for the point $(-t, t)$. We denote $(n, n)$ by $\vec{n}$. By $\mathrm{e}_{1}, \mathrm{e}_{2}$, we denote the unit vectors along the $x$ and $y$ axes respectively in the plane. To prevent cluttering due to ceiling and floor signs, we do not worry about rounding issues; our arguments are insensitive to them and remain unchanged.

2.1. Statements of results. The first result concerns right tail estimates for the exit time in stationary LPP. Let $v_{N}=\left((1-\rho)^{2} N, \rho^{2} N\right)$ be a general point on the characteristic line (see [18]) for the stationary model of density $\rho$. In the setting of Definition 2.2, define the exit time $Z^{\mathbf{0} \rightarrow v_{N}}$ to be the non-zero coordinate of the point at which the stationary geodesic from $\mathbf{0}$ to $v_{N}$ exits the coordinate axes, the convention being that the exit time is positive if the exit occurs along the $x$ axis and negative if it occurs along the $y$ axis. We prove the following estimate for the right tail of the exit time:

Theorem 2.4. There exist positive constants $N_{0}, C, c$ that depend only on $\rho \in(0,1)$ such that for all $r>0$, $N \geq N_{0}$, we have

$$
\mathbb{P}^{\rho}\left(\left|Z^{0 \rightarrow v_{N}}\right| \geq r N^{2 / 3}\right) \leq C e^{-c r^{3}} .
$$

As we already mentioned in the introduction, the above result was very recently obtained in the paper [10]. There, it was obtained by using explicit calculations for the log-moment generating function of the stationary passage time. The lower bound with the optimal exponent for the exit time is also known [17, 4]. Some estimates for the exit time along similar lines are also available in [12]. Now, we state the upper tail estimates that we obtain for the stationary last passage time along the characteristic line.

Theorem 2.5. For each fixed $\delta_{1} \in(0,1)$, there exist constants $C, c$ depending only on $\delta_{1}, \rho \in(0,1)$ such that for all $N \geq N_{0}$ and $y$ satisfying $\delta_{1} N^{2 / 3}>y>0$, we have

(1) $\mathbb{P}^{\rho}\left(G_{\text {stat }}^{1}\left(v_{N}\right)-N \geq y N^{1 / 3}\right) \leq C e^{-c y^{3 / 2}}$. 
(2) $\mathbb{P}^{\rho}\left(G_{\text {stat }}^{1}\left(v_{N}\right)-N \geq y N^{1 / 3}\right) \geq C e^{-c y^{3 / 2}}$.

We now state the lower tail estimates that we obtain for the stationary last passage time along the characteristic line.

Theorem 2.6. For any fixed $\delta_{1} \in(0,1)$, there exist constants $C, c$ depending on $\rho, \delta_{1}$ such that for all $N \geq N_{0}$ and $y$ satisfying $\delta_{1} N^{2 / 3}>y>0$, we have

(1) $\mathbb{P}^{\frac{1}{2}}\left(G_{\text {stat }}^{1}\left(v_{N}\right)-N \leq-y N^{1 / 3}\right) \geq C e^{-c y^{3}}$.

(2) $\mathbb{P}^{\rho}\left(G_{\text {stat }}^{1}\left(v_{N}\right)-N \leq-y N^{1 / 3}\right) \leq C e^{-c y^{3}}$.

Though we give matching upper and lower bounds only for $\rho=\frac{1}{2}$, the upper bound that we give for general $\rho$ is also optimal. Indeed, in the limit $N \rightarrow \infty$, the normalized passage time $\frac{G_{\text {stat }}^{1}\left(v_{N}\right)-N}{N^{1 / 3}}$ is known to converge in distribution to the Baik-Rains distribution which is known to have the tail estimates [1] that we obtain for the finite $N$ case. At this point we are unable to obtain the lower bound for general $\rho$; the reader can refer to Remark 6.6 for a discussion of the difficulties.

As we mentioned earlier, the optimal right tail estimate for the exit time, as well as the optimal upper bound estimate for the upper tail were recently obtained in the paper [10] by a different approach. It is plausible that the explicit moment generating function calculations used in [10] can also be used to obtain the results for the lower tail, but we think that it is of value to observe that these can also be obtained by comparison with the exponential LPP models with the step and flat initial conditions.

\section{TECHNiCAL IngREDIEnts}

For exponential LPP, we know that $\frac{\mathbb{E}[G(\mathbf{0}, \alpha(m, n))]}{\alpha} \rightarrow(\sqrt{m}+\sqrt{n})^{2}$ as $\alpha \rightarrow \infty[16,13]$. To reduce clutter, define $f((m, n))=(\sqrt{m}+\sqrt{n})^{2}$. Similarly, define $g(x)=\frac{x}{1-\rho}$ for $x \geq 0$ and $g(x)=-\frac{x}{\rho}$ for $x<0$. Finally, define $h(x)=\frac{\rho x^{2}}{4(1-\rho)^{3}}$ for $x \geq 0$ and $h(x)=\frac{(1-\rho) x^{2}}{4 \rho^{3}}$ for $x<0$.

The following lemma roughly says that for $\mathbf{x}$ where $1<x \ll N, \mathbb{E}^{\rho}\left[G_{\text {stat }}^{1}(\mathbf{x})\right]+\mathbb{E}^{\rho}\left[G\left(\mathbf{x}^{\uparrow}, v_{N}\right)\right] \simeq$ $\mathbb{E}^{\rho}\left[G_{\text {stat }}^{1}\left(v_{N}\right)\right]-\frac{\rho x^{2}}{4(1-\rho)^{3} N}$, where $\mathbf{x}^{\uparrow}=(x, 1)$. Note that $\mathbb{E}^{\rho}\left[G\left(\mathbf{x}^{\uparrow}, v_{N}\right)\right]=\mathbb{E}\left[G\left(\mathbf{x}^{\uparrow}, v_{N}\right)\right]$ because in the positive quadrant, the boundary representation of stationary LPP differs from exponential LPP only at the boundaries. Also note that $\mathbb{E}^{\rho}\left[G_{\text {stat }}^{1}(\mathbf{x})\right]=\frac{x}{1-\rho}$ for $x>0$ because it is a sum of $x$ independent $\exp (1-\rho)$ weights with a similar corresponding statement holding for $x<0$.

Lemma 3.1. For all $x$ with $-\rho^{2} N<x<(1-\rho)^{2} N$, we have

$$
g(x)+f\left(v_{N}-\mathbf{x}^{\uparrow}\right)=N-\frac{h(x)}{N}-N \mathcal{O}\left(\left(\frac{x}{N}\right)^{3}\right)
$$

where the $\mathcal{O}\left(\left(\frac{x}{N}\right)^{3}\right)$ is a term that is strictly positive for all $x$ in the given range.

Proof. The proof follows by plugging in the expression for $f\left(v_{N}\right)$ and doing a Taylor expansion. Note that $f\left(v_{N}\right)=N$.

The main idea used in the proof of Theorem 2.4 comes from the above lemma. Lemma 3.1 roughly shows that $\mathbb{E}^{\rho}\left[G_{\text {stat }}^{1}(\mathbf{x})+G\left(\mathbf{x}^{\uparrow}, v_{N}\right)\right]$ is about $N-C \frac{x^{2}}{N}$. Note that $\mathbb{E}^{\rho}\left[G_{\text {stat }}^{1}(\mathbf{x})+G\left(\mathbf{x}^{\uparrow}, v_{N}\right)\right]$ is the expected weight of the best up-right path from $\mathbf{0}$ to $v_{N}$ which exits at $\mathbf{x}$. On the other hand, by comparison with the point-to-point estimates for exponential LPP, we already know that $\mathbb{E}^{\rho}\left[G_{\text {stat }}^{1}\left(v_{N}\right)\right]$ is at least $N$, and we also have upper bound estimates for the lower tail. Due to the discrepancy between the means and the good concentration estimates about their respective means (Proposition 3.2 and Proposition 3.4), it is unlikely that we have $G_{\text {stat }}^{1}(\mathbf{x})+G\left(\mathbf{x}^{\uparrow}, v_{N}\right) \geq G_{\text {stat }}^{1}\left(v_{N}\right)$. When done formally, this gives us an upper bound of the probability of the exit time being exactly $\mathbf{x}$. We will finally do it for a range of $x$ simultaneously which adds technicality, but the basic idea is still the same.

One ingredient that we will use is the following point-to-point moderate deviation estimate for exponential LPP coming from [14]:

Proposition 3.2. For each $\psi>1$, there exist $C, c>0$ depending on $\psi$ such that for all $m, n$ sufficiently large with $\psi^{-1}<\frac{m}{n}<\psi$ and all $y>0$, we have the following: 
(1) $\mathbb{P}\left(G(\mathbf{0},(m, n))-(\sqrt{m}+\sqrt{n})^{2} \geq y n^{1 / 3}\right) \leq C e^{-c \min \left\{y^{3 / 2}, y n^{1 / 3}\right\}}$.

(2) $\mathbb{P}\left(G(\mathbf{0},(m, n))-(\sqrt{m}+\sqrt{n})^{2} \leq-y n^{1 / 3}\right) \leq C e^{-c y^{3}}$.

For convenience, we have taken the above specific version of the result from Theorem 4.1 in [6]. We will also need a lower bound estimate for the upper tail for point-to-point exponential LPP. It is obtained from Theorem 4 in [14] in the same way as Proposition 3.2 is obtained from using results from [14] as described in [6]. Note that Theorem 4 in [14] is stated for Hermite ensembles, but as mentioned in [14], the same technique works for the Laguerre case.

Proposition 3.3. For each $\psi>1$, there exist $C, c>0$ depending on $\psi$ such that for all $m, n$ sufficiently large with $\psi^{-1}<\frac{m}{n}<\psi$ and all $y>0$, we have the following:

$$
\mathbb{P}\left(G(\mathbf{0},(m, n))-(\sqrt{m}+\sqrt{n})^{2} \geq y n^{1 / 3}\right) \geq C e^{-c y^{3 / 2}} .
$$

Apart from using the point-to-point moderate deviation estimate, we will also be using the point-to-line estimate:

Proposition 3.4. Fix a $\rho \in(0,1)$ and $\delta_{2}>0$. Consider a line segment $\mathbb{L}_{m}(N)$ on $\{y=0\}$ with midpoint $\left(m(1-\rho)^{2} N, 0\right)$ and length $2 N^{2 / 3}$. For each $\psi \in(0,1)$, there exists $C, c>0$ (depending only on $\rho, \psi, \delta_{2}$ ) such that for all $|m|<\psi N^{1 / 3}$ and $y$ satisfying $\delta_{2} N^{2 / 3}>y>0$, we have

$$
\mathbb{P}\left(\max _{x \in \mathbb{L}_{m}(n)}\left\{G\left(\mathbf{x}^{\uparrow}, v_{N}\right)-\mathbb{E}\left[G\left(\mathbf{x}^{\uparrow}, v_{N}\right)\right]\right\}>y N^{1 / 3}\right) \leq C e^{-c y^{3 / 2}} .
$$

Proposition 3.4 is a special case of Theorem 10.5 in [9] where it is written for the more general case of parallelograms. Note that Theorem 10.5 in [9] gives an upper bound of $C e^{-c y}$ but an inspection of the proof reveals that the exponent $y$ comes from using a suboptimal point-to-point upper bound, but using the same argument with the optimal point-to-point upper bound as in Proposition 3.2 gives the correct exponent of $y^{3 / 2}$. Also, for Theorem 10.5 in [9], it a-priori appears that the vertices of the parallelogram are placed in a manner not resembling our setting, but an inspection of the proof shows that the vertices can be situated on any lines as long as the slopes of the parallelogram edges of linear length are bounded away from the coordinate directions.

In the last section, we will need the following lower bound of the lower tail probability of the point-to-line passage time in exponential LPP:

Proposition 3.5. For any constant $\delta_{2} \in(0,4)$, there exist constants $c>0, n_{0} \in \mathbb{N}$ depending on $\delta_{2}$ such that for all $n>n_{0}$ and $y \in\left(1, \delta_{2} n^{2 / 3}\right)$, we have

$$
\mathbb{P}\left(\max _{t \in \mathbb{Z}}\{G((t,-t),(n, n))\} \leq 4 n-y n^{1 / 3}\right) \geq e^{-c y^{3}} .
$$

The above proposition comes from Theorem 1.2 from [5]. In [5], the result is stated for $\delta_{2}=1$, but any $\delta_{2} \in(0,4)$ works by Theorem 2 along with the remarks at the end of the first section therein.

\section{UPPER BOUND FOR THE EXIT TIME}

We now proceed with the proof of Theorem 2.4. To begin, we bound the probability of the exit time lying in the interval $\left[r N^{2 / 3},(r+1) N^{2 / 3}\right]$ where $r$ is an integer and $-\gamma_{2} N^{2 / 3}<r<\gamma_{1} N^{2 / 3}$ for some fixed $\gamma_{1}, \gamma_{2}$ satisfying $0<\gamma_{1}<(1-\rho)^{2}$ and $0<\gamma_{2}<\rho^{2}$. We first aim to show the following intermediate result in the proof of Theorem 2.4:

Proposition 4.1. Fix positive constants $\gamma_{1}, \gamma_{2}$ such that $(1-\rho)^{2}>\gamma_{1}>0$ and $\rho^{2}>\gamma_{2}>0$. There exist constants $C, c, N_{0}$ depending on $\gamma_{1}, \gamma_{2}, \rho$ such that for all integers $r$ with $-\gamma_{2} N^{1 / 3}<r<\gamma_{1} N^{1 / 3}$ and $N \geq N_{0}$, we have

$$
\mathbb{P}^{\rho}\left(Z^{0 \rightarrow v_{N}} \in\left[r N^{2 / 3},(r+1) N^{2 / 3}\right]\right) \leq C e^{-c|r|^{3}} .
$$


We split the above probability into two parts by using a union bound as follows:

$$
\begin{aligned}
\mathbb{P}^{\rho}\left(Z^{0 \rightarrow v_{N}} \in\left[r N^{2 / 3},(r+1) N^{2 / 3}\right]\right) & =\mathbb{P}^{\rho}\left(\max _{x \in\left[r N^{2 / 3},(r+1) N^{2 / 3}\right]}\left\{G_{\text {stat }}^{1}(\mathbf{x})+G\left(\mathbf{x}^{\uparrow}, v_{N}\right)\right\} \geq G_{\text {stat }}^{1}\left(v_{N}\right)\right) \\
& \leq \mathbb{P}^{\rho}\left(G_{\text {stat }}^{1}\left(v_{N}\right) \leq \alpha\right)+\mathbb{P}^{\rho}\left(\max _{x \in\left[r N^{2 / 3},(r+1) N^{2 / 3}\right]}\left\{G_{\text {stat }}^{1}(\mathbf{x})+G\left(\mathbf{x}^{\uparrow}, v_{N}\right)\right\} \geq \alpha\right) .
\end{aligned}
$$

The above works for any $\alpha$ but to get good estimates, we need to choose $\alpha$ such that both the terms in the above expression are small. That is, $\alpha$ should be far enough from the means of both $G_{\text {stat }}^{1}\left(v_{N}\right)$ and $\max _{x \in\left[r N^{2 / 3},(r+1) N^{2 / 3}\right]}\left\{G_{\text {stat }}^{1}(\mathbf{x})+G\left(\mathbf{x}^{\uparrow}, v_{N}\right)\right\}$. Combining Lemma 3.1 with the above intuition, we will set $\alpha$ to be about $N-\frac{h\left(r N^{2 / 3}\right)}{2 N}$. Note that $\frac{x^{2}}{N}=r^{2} N^{1 / 3}$ if $x=r N^{2 / 3}$. We now bound each of the terms in (4.1). To bound the first term, we use Proposition 3.2.

Lemma 4.2. There exists $N_{0}$ such that for some positive constants $C, c$ depending on $\rho$, and for all $N>N_{0}$ and all $r$, we have that

$$
\mathbb{P}^{\rho}\left(G_{\text {stat }}^{1}\left(v_{N}\right) \leq N-r^{2} N^{1 / 3}\right) \leq C e^{-c|r|^{6}}
$$

Proof. The proof for the same statement under $\mathbb{P}(\cdot)$ instead of $\mathbb{P}^{\rho}(\cdot)$ would be a direct application of Proposition 3.2. To do it for $\mathbb{P}^{\rho}(\cdot)$, note that we have $\left\{G_{\text {stat }}^{1}\left(v_{N}\right) \leq N-r^{2} N^{1 / 3}\right\} \subseteq\left\{G\left((1,1), v_{N}\right) \leq(N-1)-\left(r^{2} N^{1 / 3}-1\right)\right\}$ which gives that

$$
\begin{aligned}
\mathbb{P}^{\rho}\left(G_{\text {stat }}^{1}\left(v_{N}\right) \leq N-r^{2} N^{1 / 3}\right) & \leq \mathbb{P}^{\rho}\left(G\left((1,1), v_{N}\right) \leq(N-1)-\left(r^{2} N^{1 / 3}-1\right)\right) \\
& =\mathbb{P}\left(G\left((1,1), v_{N}\right) \leq(N-1)-\left(r^{2} N^{1 / 3}-1\right)\right) \\
& \leq C e^{-c|r|^{6}} .
\end{aligned}
$$

Here, we used Proposition 3.2 in the last step.

Note that Lemma 3.1 says that for $x>0, \frac{x}{1-\rho}+f\left(v_{N}-\mathbf{x}^{\uparrow}\right)$ decreases as $x$ increases. To simplify the coming expressions, define $\underline{x}=r N^{2 / 3}$ and $\bar{x}=(r+1) N^{2 / 3}$ for $r \geq 0$ and $\underline{x}=(r+1) N^{2 / 3}$ and $\bar{x}=r N^{2 / 3}$ for $r<0$. All the max symbols from now till the end of the second section denote the maximum over the variable $x$ varying in the interval $\left[r N^{2 / 3},(r+1) N^{2 / 3}\right]$. For the second term in (4.1), we have

$$
\begin{aligned}
& \mathbb{P}^{\rho}\left(\max \left\{G_{\text {stat }}^{1}(\mathbf{x})+G\left(\mathbf{x}^{\uparrow}, v_{N}\right)\right\} \geq N-\frac{h(\underline{x})}{2 N}\right) \\
& \leq \mathbb{P}^{\rho}\left(\max \left\{\left(G_{\text {stat }}^{1}(\mathbf{x})-g(x)\right)+\left(G\left(\mathbf{x}^{\uparrow}, v_{N}\right)-f\left(v_{N}-\mathbf{x}^{\uparrow}\right)\right)\right\} \geq N-\frac{h(\underline{x})}{2 N}-g(\underline{x})-f\left(v_{N}-\underline{\mathbf{x}}^{\uparrow}\right)\right) \\
& =\mathbb{P}^{\rho}\left(\max \left\{\left(G_{\text {stat }}^{1}(\mathbf{x})-g(x)\right)+\left(G\left(\mathbf{x}^{\uparrow}, v_{N}\right)-f\left(v_{N}-\mathbf{x}^{\uparrow}\right)\right)\right\} \geq \frac{h(\underline{x})}{2 N}+N \mathcal{O}\left(\left(\frac{\underline{x}}{N}\right)^{3}\right)\right) \\
& \leq \mathbb{P}^{\rho}\left(\max \left\{\left(G_{\text {stat }}^{1}(\mathbf{x})-g(x)\right)+\left(G\left(\mathbf{x}^{\uparrow}, v_{N}\right)-f\left(v_{N}-\mathbf{x}^{\uparrow}\right)\right)\right\} \geq \frac{h(\underline{x})}{2 N}\right) \\
& \leq \mathbb{P}^{\rho}\left(\max \left\{\left(G_{\text {stat }}^{1}(\mathbf{x})-g(x)\right)\right\} \geq \frac{h(\underline{x})}{4 N}\right)+\mathbb{P}^{\rho}\left(\max \left\{\left(\left(G\left(\mathbf{x}^{\uparrow}, v_{N}\right)-f\left(v_{N}-\mathbf{x}^{\uparrow}\right)\right)\right\} \geq \frac{h(\underline{x})}{4 N}\right) .\right.
\end{aligned}
$$

Note that the second inequality follows because the $\mathcal{O}\left(\left(\frac{x}{N}\right)^{3}\right)$ in Lemma 3.1 is strictly positive. We again bound each of the terms in (4.2) separately. The first term is handled in the following lemma:

Lemma 4.3. For any fixed positive $\gamma_{1}, \gamma_{2}$ such that $\gamma_{1}<(1-\rho)^{2}$ and $\gamma_{2}<\rho^{2}$, there exist positive constants $N_{0}, C, c$ depending on $\rho, \gamma_{1}, \gamma_{2}$ such that for all $N>N_{0}$ and all integers $r$ such that $\gamma_{1} N^{1 / 3}>r>-\gamma_{2} N^{1 / 3}$, we have

$$
\mathbb{P}^{\rho}\left(\max _{x \in\left[r N^{2 / 3},(r+1) N^{2 / 3}\right]}\left\{G_{\text {stat }}^{1}(\mathbf{x})-g(x)\right\} \geq \frac{h(\underline{x})}{4 N}\right) \leq C e^{-c|r|^{3}} .
$$


Proof. Note that $M_{n}=G(\mathbf{0}, \mathbf{n})-\frac{n}{1-\rho}$ where $n \in\left(r N^{2 / 3},(r+1) N^{2 / 3}\right)$ is a martingale. Also note that we do the proof for the case $r \geq 0$. For negative $r$, the proof is the same, except that the martingale $M_{-n}^{\prime}=G(-\mathbf{n}, \mathbf{0})$ is used instead of $M_{n}$. Coming back to the case $r \geq 0$, on using Doob's maximal inequality for $M_{n}$, we get that for any $\lambda>0$ and $r>\frac{1}{3}$,

$$
\mathbb{P}^{\rho}\left(\max \left\{G_{\text {stat }}^{1}(\mathbf{x})-\frac{x}{1-\rho}\right\} \geq \frac{N^{1 / 3} r^{2} \rho}{16(1-\rho)^{3}}\right) \leq \frac{1}{\exp \left(\frac{\lambda r^{2} \rho}{16 \sqrt{r+1}(1-\rho)^{2}}\right)} \mathbb{E}^{\rho}\left[\exp \left(\frac{\lambda\left(G_{\text {stat }}^{1}(\overline{\mathbf{x}})-\frac{\bar{x}}{1-\rho}\right)}{\frac{\sqrt{r+1} N^{1 / 3}}{1-\rho}}\right)\right] .
$$

Using that $r>\frac{1}{3}$, we finally get that

$$
\mathbb{P}^{\rho}\left(\max \left\{G_{\text {stat }}^{1}(\mathbf{x})-\frac{x}{1-\rho}\right\} \geq \frac{N^{1 / 3} r^{2} \rho}{16(1-\rho)^{3}}\right) \leq \frac{1}{\exp \left(\frac{\lambda r^{3 / 2} \rho}{32(1-\rho)^{2}}\right)} \mathbb{E}^{\rho}\left[\exp \left(\frac{\lambda\left(G_{\text {stat }}^{1}(\overline{\mathbf{x}})-\frac{\bar{x}}{1-\rho}\right)}{\frac{\sqrt{r+1} N^{1 / 3}}{1-\rho}}\right)\right] .
$$

The above term is bounded by using that $\mathbb{E}^{\rho}\left[\exp \left(\frac{\lambda\left(G_{\text {stat }}^{1}(\overline{\mathbf{x}})-\frac{\bar{x}}{1-\rho}\right)}{\frac{\sqrt{r+1} N^{1 / 3}}{1-\rho}}\right)\right] \leq e^{C^{*} \lambda^{2}}$ for a proper choice of parameters, and some constant $C^{*}$ depending on $\gamma_{1}, \rho$. This is formally done in Lemma 4.5 which is proved using the technical Lemma 4.4; the proofs are routine and are moved to the appendix. Returning to the proof of the lemma, looking at (4.3), we choose $\lambda$ so that it minimizes $C^{*} \lambda^{2}-\frac{\lambda r^{3 / 2} \rho}{32(1-\rho)^{2}}$, that is, we choose $\lambda=\frac{r^{3 / 2} \rho}{64 C^{*}(1-\rho)^{2}}$, and the value of the above expression for this choice of $\lambda$ is $-\frac{r^{3} \rho^{2}}{2^{12} C^{*}(1-\rho)^{4}}$. Plugging in this value of $\lambda$ in (4.3), we get that for $N$ large enough, and for $\gamma_{1} N^{1 / 3}>r>\frac{1}{3} \vee r_{0}$,

$$
\mathbb{P}^{\rho}\left(\max \left\{G_{\text {stat }}^{1}(\mathbf{x})-\frac{x}{1-\rho}\right\} \geq \frac{N^{1 / 3} r^{2} \rho}{16(1-\rho)^{3}}\right) \leq e^{-\frac{r^{3} \rho^{2}}{2^{12} C^{*}(1-\rho)^{4}}} .
$$

This is what we wanted to prove. To include all $0<r \leq r_{0} \vee \frac{1}{3}$, just adjust the values of the constants $C, c$ in the statement of the lemma.

Lemma 4.4. For any constant $C^{*}$ with $C^{*}>\frac{1}{2}$, there exists a constant $\delta_{0} \in(0,1)$ depending on $C^{*}, \rho$ such that for all $0<r<(1-\rho)^{2} N^{1 / 3}, N>0$ and $\lambda>0$ satisfying $0<\lambda<\delta_{0} \sqrt{\bar{x}}$, we have

$$
\mathbb{E}^{\rho}\left[\exp \left(\frac{\lambda\left(G_{\text {stat }}^{1}(\overline{\mathbf{x}})-\frac{\bar{x}}{1-\rho}\right)}{\frac{\sqrt{r+1} N^{1 / 3}}{1-\rho}}\right)\right] \leq e^{C^{*} \lambda^{2}} .
$$

Note that $\delta_{0}$ can be chosen such that $\delta_{0} \rightarrow 1$ as $C^{*} \rightarrow \infty$.

Lemma 4.5. For any positive constant $\gamma_{1}$ such that $\gamma_{1}<(1-\rho)^{2}$, there exist positive constants $C^{*}, N_{1}, r_{0}$ depending on $\rho, \gamma_{1}$ such that for $r_{0}<r<\gamma_{1} N^{1 / 3}$ and $\lambda=\frac{r^{3 / 2} \rho}{64 C^{*}(1-\rho)^{2}}$ and $N \geq N_{1}$, we have

$$
\mathbb{E}^{\rho}\left[\exp \left(\frac{\lambda\left(G_{\text {stat }}^{1}(\overline{\mathbf{x}})-\frac{\bar{x}}{1-\rho}\right)}{\frac{\sqrt{r+1} N^{1 / 3}}{1-\rho}}\right)\right] \leq e^{C^{*} \lambda^{2}} .
$$

As we mentioned earlier, the proofs of Lemma 4.4 and Lemma 4.5 have been postponed to the appendix. We now bound the second term in (4.2), that is, we aim to show the following lemma:

Lemma 4.6. For any fixed positive $\gamma_{1}, \gamma_{2}$ with $\gamma_{1}<(1-\rho)^{2}$ and $\gamma_{2}<\rho^{2}$, there exist $N_{0}, C, c$ depending on $\rho, \gamma_{1}, \gamma_{2}$ such that for all $N>N_{0}$ and all integers $r$ with $\gamma_{1} N^{1 / 3}>r>-\gamma_{2} N^{1 / 3}$, we have that

$$
\mathbb{P}^{\rho}\left(\max _{x \in\left[r N^{2 / 3},(r+1) N^{2 / 3}\right]}\left\{G\left(\mathbf{x}^{\uparrow}, v_{N}\right)-f\left(v_{N}-\mathbf{x}^{\uparrow}\right)\right\} \geq \frac{h(\underline{x})}{4 N}\right) \leq C e^{-c|r|^{3}} .
$$

Proof. To begin, note that we only need to show the above result for $|r|>r_{0}$ for some positive constant $r_{0}$ since we can handle the case of small $r$ by adjusting the constants $C, c$. We only do it for the case $r>0$; the other case is analogous. Now, for a specific $C^{\prime}>0$, we have that

$$
\begin{aligned}
& \mathbb{P}^{\rho}\left(\max \left\{G\left(\mathbf{x}^{\uparrow}, v_{N}\right)-f\left(v_{N}-\mathbf{x}^{\uparrow}\right)\right\} \geq \frac{N^{1 / 3} r^{2} \rho}{16(1-\rho)^{3}}\right) \\
& \leq \mathbb{P}^{\rho}\left(\max \left\{G\left(\mathbf{x}^{\uparrow}, v_{N}\right)-\mathbb{E}^{\rho}\left[G\left(\mathbf{x}^{\uparrow}, v_{N}\right)\right]\right\} \geq \frac{N^{1 / 3} r^{2} \rho}{16(1-\rho)^{3}}-C^{\prime} N^{1 / 3}\right) .
\end{aligned}
$$


To get the above expression, the constant $C^{\prime}$ is chosen so that $\left|\mathbb{E}^{\rho}\left[G\left(\mathbf{x}^{\uparrow}, v_{N}\right)\right]-f\left(v_{N}-\mathbf{x}^{\uparrow}\right)\right|<C^{\prime} N^{1 / 3}$ for all $x \in\left[r N^{2 / 3},(r+1) N^{2 / 3}\right]$. Indeed, for a fixed choice of $\gamma_{1}$ and $\gamma_{2},-\gamma_{2} N^{2 / 3}<r<\gamma_{1} N^{2 / 3}$ implies that for all $z \in\left(-\gamma_{2} N^{2 / 3}, \gamma_{1} N^{2 / 3}\right)$, we have that the straight line joining $\mathbf{z}^{\uparrow}$ and $v_{N}$ has slope uniformly bounded away from 0 and $\infty$. Hence, we are in the setting of Proposition 3.2 which implies that for some constant $C^{\prime}$ (depending only on $\left.\gamma_{1}, \gamma_{2}, \rho\right)$, we have $\left|\mathbb{E}^{\rho}\left[G\left(\mathbf{x}^{\uparrow}, v_{N}\right)\right]-f\left(v_{N}-\mathbf{x}^{\uparrow}\right)\right|<C^{\prime} N^{1 / 3}$ for all $x \in\left[r N^{2 / 3},(r+1) N^{2 / 3}\right]$. Choosing $r_{0}$ large depending on $C^{\prime}$, we get that

$$
\begin{aligned}
& \mathbb{P}^{\rho}\left(\max \left\{G\left(\mathbf{x}^{\uparrow}, v_{N}\right)-\mathbb{E}^{\rho}\left[G\left(\mathbf{x}^{\uparrow}, v_{N}\right)\right]\right\} \geq \frac{N^{1 / 3} r^{2} \rho}{16(1-\rho)^{3}}-C^{\prime} N^{1 / 3}\right) \\
& \leq \mathbb{P}^{\rho}\left(\max \left\{G\left(\mathbf{x}^{\uparrow}, v_{N}\right)-\mathbb{E}^{\rho}\left[G\left(\mathbf{x}^{\uparrow}, v_{N}\right)\right]\right\} \geq \frac{N^{1 / 3} r^{2} \rho}{32(1-\rho)^{3}}\right) .
\end{aligned}
$$

To finish, just observe that the final expression fits exactly in the setting of Proposition 3.4, and note that $\left(r^{2}\right)^{3 / 2}=r^{3}$.

Proof of Proposition 4.1. By (4.1) and (4.2), we have

$$
\begin{aligned}
& \mathbb{P}^{\rho}\left(Z^{0 \rightarrow v_{N}} \in\left[r N^{2 / 3},(r+1) N^{2 / 3}\right]\right) \\
& \leq \mathbb{P}^{\rho}\left(G_{\text {stat }}^{1}\left(v_{N}\right) \leq N-\frac{h(\underline{x})}{2 N}\right)+\mathbb{P}^{\rho}\left(\max _{x \in\left[r N^{2 / 3},(r+1) N^{2 / 3}\right]}\left\{G_{\text {stat }}^{1}(\mathbf{x})+G\left(\mathbf{x}^{\uparrow}, v_{N}\right)\right\} \geq \frac{h(\underline{x})}{2 N}\right) \\
& \leq C_{1} e^{-c_{1} r^{6}}+\mathbb{P}^{\rho}\left(\max \left\{G_{\text {stat }}^{1}(\mathbf{x})-g(x)\right\} \geq \frac{h(\underline{x})}{4 N}\right)+\mathbb{P}^{\rho}\left(\max \left\{G\left(\mathbf{x}^{\uparrow}, v_{N}\right)-f\left(v_{N}-\mathbf{x}^{\uparrow}\right)\right\} \geq \frac{h(\underline{x})}{4 N}\right) \\
& \leq C_{1} e^{-c_{1} r^{6}}+C_{2} e^{-c_{2}|r|^{3}}+C_{3} e^{-c_{3}|r|^{3}} \leq C e^{-c|r|^{3}} .
\end{aligned}
$$

The first term in the fourth line was obtained by using Lemma 4.2 and the next two terms in the fourth line were obtained by using Lemma 4.3 and Lemma 4.6.

We now use Proposition 4.1 to prove Theorem 2.4.

Proof of Theorem 2.4. We will show that for all $r^{\prime}>0$ and $N$ sufficiently large,

$$
\mathbb{P}^{\rho}\left(Z^{0 \rightarrow v_{N}} \geq r^{\prime} N^{2 / 3}\right) \leq C e^{-c r^{\prime 3}} .
$$

Note that we are only doing the proof for $r^{\prime}>0$ but the proof for $r^{\prime}<0$ is the same with the role of $\gamma_{1}$ being replaced by $\gamma_{2}$. Note that we only need to worry about $r^{\prime}>r_{0}$ as we can adjust the constants to get the result for small $r^{\prime}$. For any fixed positive $\gamma_{1}$ with $\gamma_{1}<(1-\rho)^{2}$, note that

$$
\mathbb{P}^{\rho}\left(Z^{0 \rightarrow v_{N}} \geq r^{\prime} N^{2 / 3}\right) \leq \sum_{i=0}^{\gamma_{1} N^{1 / 3}-r^{\prime}} \mathbb{P}^{\rho}\left(Z^{0 \rightarrow v_{N}} \in\left[\left(r^{\prime}+i\right) N^{2 / 3},\left(r^{\prime}+i+1\right) N^{2 / 3}\right]\right)+\mathbb{P}^{\rho}\left(Z^{0 \rightarrow v_{N}} \geq \gamma_{1} N\right) .
$$

Note that the first term is present only if $r^{\prime} \leq \gamma_{1} N^{1 / 3}$. The first term involving the sum can now be bounded by using Proposition 4.1 as follows-

$$
\sum_{i=0}^{\gamma_{1} N^{1 / 3}-r^{\prime}} \mathbb{P}^{\rho}\left(Z^{0 \rightarrow v_{N}} \in\left[\left(r^{\prime}+i\right) N^{2 / 3},\left(r^{\prime}+i+1\right) N^{2 / 3}\right]\right) \leq \sum_{i=0}^{\infty} C_{1} e^{-c_{1}\left(r^{\prime}+i\right)^{3}} \leq C_{1}^{\prime} e^{-c_{1}^{\prime} r^{\prime 3}} .
$$

Here $C_{1}^{\prime}, c_{1}^{\prime}$ are constants depending on $\gamma_{1}, \rho$. We will be choosing a specific value of $\gamma_{1}$ later in the argument. It now remains to bound the second term in (4.7). It turns out that the far end of the tail is easy to bound by a different argument. Note that we have the following crude estimate:

$$
\mathbb{P}^{\rho}\left(Z^{\mathbf{0} \rightarrow v_{N}} \geq \gamma_{1} N\right) \leq \mathbb{P}^{\rho}\left(G\left(\mathbf{0},\left((1-\rho)^{2} N, 0\right)\right)+G\left(\left(\gamma_{1} N, 1\right), v_{N}\right)>G_{\text {stat }}^{1}\left(v_{N}\right)\right) .
$$

The same strategy used in the first term above works again if we can choose $\gamma_{1}$ so that $\mathbb{E}^{\rho}\left[G_{\text {stat }}^{1}(((1-\right.$ $\left.\left.\left.\rho)^{2} N, 0\right)\right)\right]+f\left(v_{N}-\left(\gamma_{1} N, 1\right)\right)$ is at most $N-\beta N$ for some $0<\beta<1$ depending on $\rho$. Noting that $\mathbb{E}^{\rho}\left[G_{\text {stat }}^{1}\left(\left((1-\rho)^{2} N, 0\right)\right)\right]=(1-\rho) N$, we have

$$
\mathbb{E}^{\rho}\left[G_{\text {stat }}^{1}\left(\left((1-\rho)^{2} N, 0\right)\right)\right]+f\left(v_{N}-\left(\gamma_{1} N, 1\right)\right)=N\left((1-\rho)+\left(\sqrt{\rho^{2}-\frac{1}{N^{2}}}+(1-\rho) \sqrt{1-\frac{\gamma_{1}}{(1-\rho)^{2}}}\right)^{2}\right) .
$$


At $\gamma_{1}=(1-\rho)^{2}$, the coefficient of $N$ is the above expression is at most $(1-\rho)+\rho^{2}<(1-\rho)+\rho=1$. Hence, by continuity, we can choose $\gamma_{1}$ sufficiently close to $(1-\rho)^{2}$ and obtain a positive value of $\beta$ as needed. Thus, we now have for all $N$ large enough,

$$
\mathbb{E}^{\rho}\left[G_{\text {stat }}^{1}\left(\left((1-\rho)^{2} N, 0\right)\right)\right]+f\left(v_{N}-\left(\gamma_{1} N, 1\right)\right) \leq N-\beta N .
$$

Hence, by using (4.9) along with (4.8), we finally have that

$$
\begin{aligned}
& \mathbb{P}^{\rho}\left(Z^{0 \rightarrow v_{N}} \geq \gamma_{1} N\right) \\
& \leq \mathbb{P}^{\rho}\left(G_{\text {stat }}^{1}\left(v_{N}\right)<N-\frac{\beta N}{2}\right)+\mathbb{P}^{\rho}\left(G_{\text {stat }}^{1}\left(\left((1-\rho)^{2} N, 0\right)\right)-(1-\rho) N>\frac{\beta N}{4}\right) \\
& +\mathbb{P}^{\rho}\left(G\left(\left(\gamma_{1} N, 1\right), v_{N}\right)-f\left(v_{N}-\left(\gamma_{1} N, 1\right)\right)>\frac{\beta N}{4}\right) .
\end{aligned}
$$

We can now repeat the arguments in Lemma 4.2 and Lemma 4.6 to bound the first and third terms. Indeed, the arguments are only made easier because there is no max involved. Note that the point-to-line estimateProposition 3.4 used in Lemma 4.6 is now substituted with the point-to-point estimate- Proposition 3.2. The second term is bounded by using exponential concentration for sums of i.i.d. random variables. Hence, for large enough $N$, we have

$$
\mathbb{P}^{\rho}\left(Z^{0 \rightarrow v_{N}} \geq \gamma_{1} N\right) \leq C_{4} e^{-c_{4} N} .
$$

To finish the proof, we go back to (4.7). For the case $r^{\prime} \leq \gamma_{1} N^{1 / 3}$, we have

$$
\mathbb{P}^{\rho}\left(Z^{0 \rightarrow v_{N}} \geq r^{\prime} N^{2 / 3}\right) \leq C_{1}^{\prime} e^{-c_{1}^{\prime} r^{\prime 3}}+C_{4} e^{-c_{4} N} \leq C_{5} e^{-c_{5} r^{\prime 3}} .
$$

For the case $r^{\prime}>\gamma_{1} N^{1 / 3}$, from (4.7), we have that

$$
\mathbb{P}^{\rho}\left(Z^{0 \rightarrow v_{N}} \geq r^{\prime} N^{2 / 3}\right) \leq C_{4} e^{-c_{4} N} \leq C e^{-c r^{\prime 3}} .
$$

Note that the last inequality follows because we can restrict to $r^{\prime} \leq(1-\rho)^{2} N^{1 / 3}$ because Theorem 2.4 is vacuously true for the case $r^{\prime}>(1-\rho)^{2} N^{1 / 3}$. Indeed, $Z^{0 \rightarrow v_{N}}$ is deterministically smaller than $(1-\rho)^{2} N$.

\section{UPPER TAIL ESTIMATES}

The proof is along the same lines as the proof of Theorem 2.4 in the previous section; indeed, some ingredients are already proven implicitly in the last section. The proof of (2) in Theorem 2.5 is straightforward by comparison to the point-to-point LPP estimates and we prove it now.

Proof of (2) in Theorem 2.5. Note that

$$
\begin{aligned}
\mathbb{P}^{\rho}\left(G_{\text {stat }}^{1}\left(v_{N}\right)-N \geq y N^{1 / 3}\right) & \geq \mathbb{P}\left(G\left((1,1), v_{N}\right)-N \geq y N^{1 / 3}\right) \\
& =\mathbb{P}\left(G\left((1,1), v_{N}\right)-(N-1) \geq y N^{1 / 3}+1\right) .
\end{aligned}
$$

Now, note that we are in the setting of Proposition 3.3 which gives the result immediately.

We now give a series of intermediate results with the aim of proving Theorem 2.5.

Proposition 5.1. For each fixed $\delta_{1} \in(0,1)$ and $\gamma_{1}, \gamma_{2}$ such that $(1-\rho)^{2}>\gamma_{1}>0$ and $\rho^{2}>\gamma_{2}>0$, there exist positive constants $C, c, N_{0}$ depending on $\rho, \gamma_{1}, \gamma_{2}, \delta_{1}$ such that for all $N \geq N_{0}$ and $y$ satisfying $0<y<\delta_{1} N^{2 / 3}$, we have

(1) $\mathbb{P}^{\rho}\left(\max _{x \in\left[1, \gamma_{1} N\right]}\left\{G_{\text {stat }}^{1}(\mathbf{x})+G\left(\mathbf{x}^{\uparrow}, v_{N}\right)\right\}>N+y N^{1 / 3}\right) \leq C e^{-c y^{3 / 2}}$.

(2) $\mathbb{P}^{\rho}\left(\max _{x \in\left[-\gamma_{2} N,-1\right]}\left\{G_{\text {stat }}^{1}(\mathbf{x})+G\left(\mathbf{x}^{\uparrow}, v_{N}\right)\right\}>N+y N^{1 / 3}\right) \leq C e^{-c y^{3 / 2}}$.

Proof. Note that we can prove the proposition for all $y>y_{0}$ for some $y_{0}$ depending only on $\rho$ and then adjust $C$ and $c$ to account for $y \leq y_{0}$. We will only prove the first part of the proposition; the proof of the 
second part is analogous. As we did in the first section, we first control the maximum in an interval of width $N^{2 / 3}$. Following (4.2), for any $0<r<\gamma_{1} N^{1 / 3}$, where $\gamma_{1}, \gamma_{2}$ will be fixed later, write

$$
\begin{aligned}
& \mathbb{P}^{\rho}\left(\max _{x \in\left[r N^{2 / 3},(r+1) N^{2 / 3}\right]}\left\{G_{\text {stat }}^{1}(\mathbf{x})+G\left(\mathbf{x}^{\uparrow}, v_{N}\right)\right\}>N+y N^{1 / 3}\right) \\
& \leq \mathbb{P}^{\rho}\left(\max \left\{G_{\text {stat }}^{1}(\mathbf{x})-\frac{x}{1-\rho}\right\}>\frac{y N^{1 / 3}}{2}+\frac{N^{1 / 3} r^{2} \rho}{16(1-\rho)^{3}}\right) \\
& +\mathbb{P}^{\rho}\left(\max \left\{G\left(\mathbf{x}^{\uparrow}, v_{N}\right)-f\left(v_{N}-\mathbf{x}^{\uparrow}\right)\right\}>\frac{y N^{1 / 3}}{2}+\frac{N^{1 / 3} r^{2} \rho}{16(1-\rho)^{3}}\right) .
\end{aligned}
$$

On repeating the proof of Lemma 4.6, we get that for all $0<r<\gamma_{1} N^{2 / 3}$, we have

$$
\mathbb{P}^{\rho}\left(\max \left\{G\left(\mathbf{x}^{\uparrow}, v_{N}\right)-f\left(v_{N}-\mathbf{x}^{\uparrow}\right)\right\}>\frac{y N^{1 / 3}}{2}+\frac{N^{1 / 3} r^{2} \rho}{16(1-\rho)^{3}}\right) \leq C_{1} e^{-\left(\frac{y}{2}+\frac{r^{2} \rho}{16(1-\rho)^{3}}-C^{\prime}\right)^{3 / 2}} .
$$

In the above expression $C^{\prime}$ comes from the proof of Lemma 4.6. This handles the second term. We will now bound the first term for all $y$ such that $y<\delta_{1} N^{2 / 3}$ by using the technique in Lemma 4.3. We have

$\mathbb{P}^{\rho}\left(\max \left\{G_{\text {stat }}^{1}(\mathbf{x})-\frac{x}{1-\rho}\right\}>\frac{y N^{1 / 3}}{2}+\frac{N^{1 / 3} r^{2} \rho}{16(1-\rho)^{3}}\right) \leq \frac{1}{\exp \left(\frac{\lambda r^{2} \rho}{16 \sqrt{r+1}(1-\rho)^{2}}+\frac{\lambda y(1-\rho)}{2 \sqrt{r+1}}\right)} \mathbb{E}^{\rho}\left[\exp \left(\frac{\lambda\left(G_{\text {stat }}^{1}(\overline{\mathbf{x}})-\frac{\bar{x}}{1-\rho}\right)}{\frac{\sqrt{r+1} N^{1 / 3}}{1-\rho}}\right)\right]$.

Since we have assumed that $y<\delta_{1} N^{2 / 3}$, we have that $\sqrt{r+1} \sqrt{y}<\delta_{1} \sqrt{\bar{x}}$. Hence, by Lemma 4.4, with $C^{*}$ such that $\delta_{0}\left(C^{*}\right) \geq \delta_{1}$, we can choose $\lambda=\sqrt{r+1} \sqrt{y}$ to finally get that for all $0<r<\gamma_{1} N^{2 / 3}$,

$$
\begin{aligned}
\mathbb{P}^{\rho}\left(\max \left\{G_{\text {stat }}^{1}(\mathbf{x})-\frac{x}{1-\rho}\right\}>\frac{y N^{1 / 3}}{2}+\frac{N^{1 / 3} r^{2} \rho}{16(1-\rho)^{3}}\right) & \leq \exp \left\{C^{*}(r+1) y-\left(\frac{\sqrt{y} r^{2} \rho}{16(1-\rho)^{2}}+\frac{y^{3 / 2}(1-\rho)}{2}\right)\right\} \\
& =\exp \left\{C^{*}\left((r+1) y-c_{3} \sqrt{y} r^{2}-c_{4} y^{3 / 2}\right)\right\} \\
& =\exp \left\{C^{*}\left(-c_{3} \sqrt{y}\left(\left(r-\frac{\sqrt{y}}{2 c_{3}}\right)^{2}-\frac{y}{4 c_{3}^{2}}-\frac{\sqrt{y}}{c_{3}}\right)-c_{4} y^{3 / 2}\right)\right\} .
\end{aligned}
$$

We will be using the above expression for the case $r<\alpha \sqrt{y}$, where $\alpha$ will be chosen later. For the case $r \geq \alpha \sqrt{y}$, we choose a different $\lambda$ in (5.3). Note that from proof of Lemma 4.5, $\frac{r^{2}}{32 C^{* *} \sqrt{r+1}(1-\rho)^{2}} \times \frac{1}{\sqrt{\bar{x}}}$ is smaller than $\delta_{0}\left(C^{* *}\right)$ (where $\delta_{0}$ comes from Lemma 4.4) if $y_{0}, N_{0}, C^{* *}$ are chosen properly. Hence, we can choose $\lambda=\frac{r^{2}}{32 C^{* *} \sqrt{r+1}(1-\rho)^{2}}$, and this gives that for $r \geq \alpha \sqrt{y}$, (increasing $y_{0}$ if necessary)

$$
\mathbb{P}^{\rho}\left(\max \left\{G_{\text {stat }}^{1}(\mathbf{x})-\frac{x}{1-\rho}\right\}>\frac{y N^{1 / 3}}{2}+\frac{N^{1 / 3} r^{2} \rho}{32(1-\rho)^{3}}\right) \leq \exp \left\{-\frac{r^{3} \rho}{2^{12} C^{* *}}(1-\rho)^{2}-\frac{r y}{2^{7}(1-\rho) C^{* *}}\right\} .
$$

Finally, we piece this together to prove Proposition 5.1. Observe that

$$
\begin{aligned}
& \mathbb{P}^{\rho}\left(\max _{x \in\left[1, \gamma_{1} N\right]}\left\{G_{\text {stat }}^{1}(\mathbf{x})+G\left(\mathbf{x}^{\uparrow}, v_{N}\right)\right\}>N+y N^{1 / 3}\right) \\
& \leq \sum_{r=0}^{\gamma_{1} N^{1 / 3}-1} \mathbb{P}^{\rho}\left(\max _{x \in\left[r N^{2 / 3},(r+1) N^{2 / 3}\right]}\left\{G_{\text {stat }}^{1}(\mathbf{x})+G\left(\mathbf{x}^{\uparrow}, v_{N}\right)\right\}>N+y N^{1 / 3}\right) .
\end{aligned}
$$

Note that each term in the above equation was split into two terms. The first term was bounded in (5.2), and note that by possibly increasing $y_{0}$, we have

$$
\sum_{r=0}^{\infty} C_{1} e^{-\left(\frac{y}{2}+\frac{r^{2} \rho}{16(1-\rho)^{3}}-C^{\prime}\right)^{3 / 2}} \leq C_{1}^{\prime} e^{-c_{1}^{\prime} y^{3 / 2}} .
$$

This handles the contribution to the sum coming from the first term. For the second term, we obtained two different bounds depending on the value of $r$. Choose $\alpha>0$ such that $0<\alpha<\frac{1}{2 c_{3}}$ and

$$
-c_{3}\left(\left(\alpha-\frac{1}{2 c_{3}}\right)^{2}-\frac{1}{4 c_{3}^{2}}\right)-c_{4}<0 .
$$


Now, note that

$$
\sum_{r=0}^{\alpha \sqrt{y}} \exp \left\{C^{*}\left(-c_{3} \sqrt{y}\left(\left(r-\frac{\sqrt{y}}{2 c_{3}}\right)^{2}-\frac{y}{4 c_{3}^{2}}-\frac{\sqrt{y}}{c_{3}}\right)-c_{4} y^{3 / 2}\right)\right\} \leq C_{2}^{\prime} e^{-c_{2}^{\prime} y^{3 / 2}} .
$$

Finally, also note that

$$
\sum_{r=\alpha \sqrt{y}}^{\infty} \exp \left\{-\frac{r^{3} \rho}{2^{12} C^{* *}}(1-\rho)^{2}-\frac{r y}{2^{7}(1-\rho) C^{* *}}\right\} \leq C_{3}^{\prime} e^{-c_{3}^{\prime} y^{3 / 2}} .
$$

Thus we finally have that

$$
\mathbb{P}^{\rho}\left(\max _{x \in\left[1, \gamma_{1} N\right]}\left\{G_{\text {stat }}^{1}(\mathbf{x})+G\left(\mathbf{x}^{\uparrow}, v_{N}\right)\right\}>N+y N^{1 / 3}\right) \leq C_{1}^{\prime} e^{-c_{1}^{\prime} y^{3 / 2}}+C_{2}^{\prime} e^{-c_{2}^{\prime} y^{3 / 2}}+C_{3}^{\prime} e^{-c_{3}^{\prime} y^{3 / 2}} \leq C e^{-c y^{3 / 2}} .
$$

This finishes the proof of the proposition.

Proof of (1) in Theorem 2.5. Fix any positive $\gamma_{1}, \gamma_{2}$ such that $\gamma_{1}<(1-\rho)^{2}$ and $\gamma_{2}<\rho^{2}$. Observe that

$$
\begin{aligned}
\mathbb{P}^{\rho}\left(G_{\text {stat }}^{1}\left(v_{N}\right)-N \geq y N^{1 / 3}\right) & \leq \mathbb{P}^{\rho}\left(\max _{x \in\left[1, \gamma_{1} N\right]}\left\{G_{\text {stat }}^{1}(\mathbf{x})+G\left(\mathbf{x}^{\uparrow}, v_{N}\right)\right\}>N+y N^{1 / 3}\right) \\
& +\mathbb{P}^{\rho}\left(\max _{x \in\left[-\gamma_{2} N,-1\right]}\left\{G_{\text {stat }}^{1}(\mathbf{x})+G\left(\mathbf{x}^{\uparrow}, v_{N}\right)\right\}>N+y N^{1 / 3}\right)+\mathbb{P}^{\rho}\left(\left|Z^{0 \rightarrow v_{N}}\right| \geq\left(\gamma_{1} \wedge \gamma_{2}\right) N\right) \\
& \leq C_{4}^{\prime} e^{-c_{4}^{\prime} y^{3 / 2}}+C_{5}^{\prime} e^{-c_{5}^{\prime} y^{3 / 2}}+C_{6}^{\prime} e^{-c_{6}^{\prime} N^{3}} \\
& \leq C e^{-c y^{3 / 2}} .
\end{aligned}
$$

The first inequality is a straightforward union bound. To get the second inequality, we have used Proposition 5.1 and Theorem 2.4 respectively. To get the final inequality, we have used that $y \leq \delta_{1} N^{2 / 3}$.

\section{Lower tail estimates}

The proof of (2) in Theorem 2.6 is straightforward by comparison to the point-to-point estimates.

Proof of (2) in Theorem 2.6. The proof is exactly the same as the proof of Lemma 4.2. One just needs to replace $r^{2}$ in the statement of Lemma 4.2 by $y$ and then reproduce the proof verbatim.

We now come to the proof of (1) in Theorem 2.6. For the remainder of the section, we will be working with $\rho=\frac{1}{2}$. We will now use the point-to-line representation of the stationary model. This helps us set up for a direct application of Proposition 3.5 which gives us the required lower bound. As we mentioned earlier, the two representations of the stationary model are equivalent, and this can be shown by coupling both the representations to a separate boundary representation model. The coupling is described in detail in the appendix, and the following proposition follows directly from the coupling:

Proposition 6.1. For $v \in \mathbb{Z}_{\geq 0}^{2}$, the random variables $\left\{G_{\text {stat }}^{1}(v)\right\}$ have the same joint distribution as the random variables $\left\{G_{\text {stat }}^{2}(v)\right\}$.

Recall the notation $\overline{\mathrm{P}}^{\rho}(\cdot)$ for the probabilities in the point-to-line stationary model. To be able to reduce a calculation in the point-to-line representation to one in the boundary representation, we will need the following result which is proved by the coupling argument in the appendix:

Proposition 6.2. In the point-to-line stationary representation, consider the non zero coordinate $q_{N}$ of the point where the stationary geodesic to $v_{N}$ last meets the coordinate axes, the convention being that $q_{N}$ is positive if the point is on the $x$ axis and is negative otherwise. Then we have that the distribution of $q_{N}$ is the same as the distribution of the exit time in the boundary representation. That is,

$$
q_{N} \stackrel{\mathcal{D}}{=} Z^{0 \rightarrow v_{N}}
$$

where the distribution of the former is considered under $\overline{\mathbb{P}}^{\rho}(\cdot)$ and the latter under $\mathbb{P}^{\rho}(\cdot)$.

Coming back to (1) in Theorem 2.6, by invoking Proposition 6.1, we equivalently need to show the following: 
Theorem 6.3. For any fixed $\delta_{1} \in(0,1)$, there exist constants $C, c$ depending on $\delta_{1}$ such that for all $N \geq N_{0}$ and for all $y$ such that $\delta_{1} N^{2 / 3}>y>0$, we have

$$
\overline{\mathbb{P}}^{\frac{1}{2}}\left(G_{\text {stat }}^{2}\left(v_{N}\right)-N \leq-y N^{1 / 3}\right) \geq C e^{-c y^{3}} .
$$

Note that for the point-to-line representation, we have the following analogue of Lemma 3.1:

Lemma 6.4. If $-\frac{N}{4}<t<\frac{N}{4}$, we have that

$$
f\left(v_{N}-(t,-t)\right)=N-4 \frac{t^{2}}{N}-N \mathcal{O}\left(\left(\frac{t}{N}\right)^{4}\right),
$$

where the $\mathcal{O}\left(\left(\frac{t}{N}\right)^{4}\right)$ is a term that is strictly positive for all $t$ in the given range.

Proof. The proof is a straightforward computation of the Taylor expansion of $\left(\sqrt{\frac{N}{4}-t}+\sqrt{\frac{N}{4}+t}\right)^{2}$.

The term $f\left(v_{N}-(t,-t)\right)$ in Lemma 6.4 can be heuristically thought of as the expected passage time from 0 to $v_{N}$ if one only maximises over paths which leave the line $\{x+y=0\}$ at the point $(t,-t)$. Indeed, there is no term here analogous to $g(x)$ in Lemma 3.1 because each random variable on the boundary line $\{x+y=0\}$ has mean 0 for the case $\rho=\frac{1}{2}$. Recall that we use the notation $\mathfrak{t}$ for $(-t, t)$. We split the proof of Theorem 6.3 into two cases- the proof for the case $y \geq \frac{1}{2} N^{1 / 6}$ is completed in Proposition 6.5 whereas the case $y<\frac{1}{2} N^{1 / 6}$ requires an extra argument.

Proposition 6.5. For $\delta_{1} \in(0,1)$, there exist positive constants $C, c, N_{0}, y_{0}$ depending on $\delta_{1}$ such that for all $N \geq N_{0}$ and $y_{0}<y<\delta_{1} N^{2 / 3}$, we have

$$
\overline{\mathbb{P}}^{\frac{1}{2}}\left(\max _{t \in\left[-\left(y^{2} N^{2 / 3} \wedge \frac{N}{4}\right), y^{2} N^{2 / 3} \wedge \frac{N}{4}\right]}\left\{T(\mathfrak{t})+G\left(\mathfrak{t}, v_{N}\right)\right\}<N-y N^{1 / 3}\right) \geq C e^{-c y^{3}} .
$$

Proof. Consider the case $y^{2} N^{2 / 3}<\frac{N}{4}$ for now. Recall the notation $G_{0}(\cdot)$ from the statement after (2.1). By increasing $y_{0}$ if necessary, we have the following:

$$
\begin{aligned}
& \overline{\mathbb{P}}^{\frac{1}{2}}\left(\max _{t \in\left[-y^{2} N^{2 / 3}, y^{2} N^{2 / 3}\right]}\left\{T(\mathfrak{t})+G\left(\mathfrak{t}, v_{N}\right)\right\}<N-y N^{1 / 3}\right) \\
& \geq \overline{\mathbb{P}}^{\frac{1}{2}}\left(\left\{\max _{t \in\left[-y^{2} N^{2 / 3}, y^{2} N^{2 / 3}\right]}\{T(\mathfrak{t})\}<\left(1-\delta_{1}\right) y N^{1 / 3}\right\} \bigcap\left\{\max _{t \in\left[-y^{2} N^{2 / 3}, y^{2} N^{2 / 3}\right]}\left\{G\left(\mathfrak{t}, v_{N}\right)\right\}<N-y\left(2-\delta_{1}\right) N^{1 / 3}\right\}\right) \\
& =\overline{\mathbb{P}}^{\frac{1}{2}}\left(\max _{t \in\left[-y^{2} N^{2 / 3}, y^{2} N^{2 / 3}\right]}\{T(\mathfrak{t})\}<\left(1-\delta_{1}\right) y N^{1 / 3}\right) \overline{\mathbb{P}}^{\frac{1}{2}}\left(\max _{t \in\left[-y^{2} N^{2 / 3}, y^{2} N^{2 / 3}\right]}\left\{G\left(\mathfrak{t}, v_{N}\right)\right\}<N-y\left(2-\delta_{1}\right) N^{1 / 3}\right) \\
& \geq \overline{\mathbb{P}}^{\frac{1}{2}}\left(\max _{t \in\left[-y^{2} N^{2 / 3}, y^{2} N^{2 / 3}\right]}\{T(\mathfrak{t})\}<\left(1-\delta_{1}\right) y N^{1 / 3}\right) \mathbb{P}\left(G_{0}\left(v_{N}\right)<N-y\left(2-\delta_{1}\right) N^{1 / 3}\right) .
\end{aligned}
$$

The first equality in the above series of expressions follows because of the independence of the boundary and non-boundary random variables. Note that the first term in (6.1) can be bounded as follows

$$
\begin{aligned}
\overline{\mathbb{P}}^{\frac{1}{2}}\left(\max _{t \in\left[-y^{2} N^{2 / 3}, y^{2} N^{2 / 3}\right]}\{T(\mathfrak{t})\}<\left(1-\delta_{1}\right) y N^{1 / 3}\right) & =\overline{\mathbb{P}}^{\frac{1}{2}}\left(\max _{t \in\left[-y^{2} N^{2 / 3}, y^{2} N^{2 / 3}\right]}\left\{\frac{T(\mathfrak{t})}{\sqrt{y^{2} N^{2 / 3}}}\right\}<1-\delta_{1}\right) \\
& \rightarrow \mathbf{P}\left(\max _{t \in[-1,1]}\left\{B_{t}\right\}<1-\delta_{1}\right)>c_{1}>0 \text { as } N \rightarrow \infty .
\end{aligned}
$$

The last line in the above expression follows by Donsker's theorem as under $\mathbf{P}, B_{t}$ has the law of the two-sided standard Brownian motion started from 0. Note that the final term is some constant and has no dependence on $y$ or $N$, and the estimate is uniform as $y>y_{0}>0$. Finally, to prove the proposition, we need to handle the second term in (6.1), and we will be using Proposition 3.5 with $\delta_{2}=4 \delta_{1}\left(2-\delta_{1}\right)$ for this purpose. Indeed, Proposition 3.5 immediately implies the following for all large $N$ :

$$
\mathbb{P}\left(G_{0}\left(v_{N}\right)<N-y\left(2-\delta_{1}\right) N^{1 / 3}\right) \geq C_{2} e^{-c_{2} y^{3}} .
$$


Note that we needed to restrict to the case $\rho=\frac{1}{2}$ to use Proposition 3.5. On combining (6.2) and (6.3) by using (6.1), we have what we needed, namely

$$
\overline{\mathbb{P}}^{\frac{1}{2}}\left(\max _{t \in\left[-y^{2} N^{2 / 3}, y^{2} N^{2 / 3}\right]}\left\{T(\mathfrak{t})+G\left(\mathfrak{t}, v_{N}\right)\right\}<N-y N^{1 / 3}\right) \geq C e^{-c y^{3}} .
$$

Note that the case $y^{2} N^{2 / 3} \geq \frac{N}{4}$ follows from the above because when we lower bound the term

$$
\overline{\mathbb{P}}^{\frac{1}{2}}\left(\max _{t \in\left[-\frac{N}{4}, \frac{N}{4}\right]}\left\{T(\mathfrak{t})+G\left(\mathfrak{t}, v_{N}\right)\right\}<N-y N^{1 / 3}\right)
$$

by using the method in (6.1), then the bound in (6.3) stays the same, whereas the bound in (6.2) only becomes better. Indeed for $y^{2} N^{2 / 3} \geq \frac{N}{4}$, we have that

$$
\overline{\mathbb{P}}^{\frac{1}{2}}\left(\max _{t \in\left[-\frac{N}{4}, \frac{N}{4}\right]}\{T(\mathfrak{t})\}<\left(1-\delta_{1}\right) y N^{1 / 3}\right) \geq \overline{\mathbb{P}}^{\frac{1}{2}}\left(\max _{t \in\left[-\frac{N}{4}, \frac{N}{4}\right]}\left\{\frac{T(\mathfrak{t})}{\sqrt{N / 4}}\right\}<1-\delta_{1}\right),
$$

and the result follows by using Donsker's theorem as in (6.2).

Remark 6.6. The same approach does not work directly for general $\rho$ because for $\rho \neq \frac{1}{2}$, the weight function $T$ on the line $\{x+y=0\}$ in the point-to-line representation is a random walk with non-zero drift which causes the Brownian estimate in (6.1) to no longer work. To use the same approach for general $\rho$, one would need to obtain a point-to-line estimate, similar to Proposition 3.5, adjusted to included the drift term on the line.

We now complete the proof of Theorem 6.3.

Proof of Theorem 6.3. Note that we already proved the result for the case $y^{2} \geq \frac{N^{1 / 3}}{4}$ in Proposition 6.5, so we restrict to the case $y^{2}<\frac{N^{1 / 3}}{4}$. Also, the case $0<y \leq y_{0}$ can be handled by adjusting $C, c$. For the point-to-line representation, let $A_{1}$ denote the event that the stationary geodesic to $v_{N}$ does not intersect the line segment $\left\{\mathfrak{t}: t \in\left[-y^{2} N^{2 / 3}, y^{2} N^{2 / 3}\right]\right\}$. Similarly, let $A_{2}$ the event that the stationary geodesic to $v_{N}$ does not intersect the region $\left\{\mathbf{x}: x \in\left[-y^{2} N^{2 / 3}, y^{2} N^{2 / 3}\right]\right\}$. Observe that we have

$$
\begin{aligned}
\overline{\mathbb{P}}^{\frac{1}{2}}\left(G_{\text {stat }}^{2}\left(v_{N}\right)-N \leq-y N^{1 / 3}\right) & \geq \overline{\mathbb{P}}^{\frac{1}{2}}\left(\max _{t \in\left[-y^{2} N^{2 / 3}, y^{2} N^{2 / 3}\right]}\left\{T(\mathfrak{t})+G\left(\mathfrak{t}, v_{N}\right)\right\}<N-y N^{1 / 3}\right)-\overline{\mathbb{P}}^{\frac{1}{2}}\left(A_{1}\right) \\
& \geq C_{1} e^{-c_{1} y^{3}}-\overline{\mathbb{P}}^{\frac{1}{2}}\left(A_{2}\right) \\
& =C_{1} e^{-c_{1} y^{3}}-\mathbb{P}^{\frac{1}{2}}\left(\left|Z^{0 \rightarrow v_{N}}\right| \geq y^{2} N^{2 / 3}\right) \\
& \geq C_{1} e^{-c_{1} y^{3}}-C_{2} e^{-c_{2} y^{6}} \\
& \geq C e^{-c y^{3}}
\end{aligned}
$$

The first inequality is a simple union bound. The second inequality follows by observing that $A_{1} \subseteq A_{2}$ along with applying Proposition 6.5. The first equality follows by applying Proposition 6.2, and the third inequality in an immediate application of Theorem 2.4.

\section{REFERENCES}

[1] Jinho Baik and Eric M. Rains. Limiting distributions for a polynuclear growth model with external sources. Journal of Statistical Physics, 100:523-541, 2000.

[2] Márton Bálazs, Ofer Busani, and Timo Seppäläinen. Non-existence of bi-infinite geodesics in the exponential corner growth model. Preprint, arXiv:1909.06883.

[3] Márton Bálazs, Eric Cator, and Timo Seppäläinen. Cube root fluctuations for the corner growth model associated to the exclusion process. Electron. J. Probab., 11:1094-1132, 2006.

[4] Márton Balázs and Timo Seppäläinen. Order of current variance and diffusivity in the asymmetric simple exclusion process. Ann. Math. (2), 171(2):1237-1265, 2010.

[5] Riddhipratim Basu, Shirshendu Ganguly, Milind Hegde, and Manjunath Krishnapur. Lower deviations in $\beta$-ensemebles and law of iterated logarithm in last passage percolation. Preprint arXiv 1909.01333v1.

[6] Riddhipratim Basu, Shirshendu Ganguly, and Lingfu Zhang. Temporal correlation in last passage percolation with flat initial condition via Brownian comparison. Preprint arXiv 1912.04891v1. 
[7] Riddhipratim Basu, Christopher Hoffman, and Allan Sly. Nonexistence of bigeodesics in integrable models of last passage percolation. Preprint, arXiv:1811.04908.

[8] Riddhipratim Basu, Sourav Sarkar, and Allan Sly. Coalescence of geodesics in exactly solvable models of last passage percolation. Journal of Mathematical Physics, 60(9):093301, 2019.

[9] Riddhipratim Basu, Vladas Sidoravicius, and Allan Sly. Last passage percolation with a defect line and the solution of the Slow Bond Problem. Preprint arXiv 1408.3464.

[10] Elnur Emrah, Chrishtopher Janjigian, and Timo Seppäläinen. Right-tail moderate deviations in the exponential last-passage percolation. Preprint, arXiv:2004.04285v1.

[11] Patrik L. Ferrari and Alessandra Occelli. Time-time covariance for last passage percolation with generic initial profile. Mathematical Physics, Analysis and Geometry, 22:1-33, 2018.

[12] Patrik L. Ferrari and Alessandra Occelli. Universality of the GOE Tracy-Widom distribution for TASEP with arbitrary particle density. Electron. J. Probab., 23:24 pp., 2018.

[13] Kurt Johansson. Shape fluctuations and random matrices. Communications in Mathematical Physics, 209(2):437-476, 2000 .

[14] Michel Ledoux and Brian Rider. Small deviations for beta ensembles. Electron. J. Probab., 15:1319-1343, 2010.

[15] Leandro P. R. Pimentel. Duality between coalescence times and exit points in last-passage percolation models. Ann. Probab., 44(5):3187-3206, 2016.

[16] H. Rost. Noniequilibrium behaviour of a many particle process: Density profile and local equi- libria. Zeitschrift $f$. Warsch. Verw. Gebiete, 58(1):41-53, 1981.

[17] Timo Seppäläinen. Variational formulas, Busemann functions, and fluctuation exponents for the corner growth model with exponential weights. 092017.

[18] Timo Seppäläinen and Xiao Shen. Coalescence estimates for the corner growth model with exponential weights. Preprint, arXiv:1911.03792v1.

\section{APPENDIX}

We first give the proof of Lemma 4.4 and Lemma 4.5.

Proof of Lemma 4.4. Using the explicit form of the moment generating function for the exponential distribution, we have that

$$
\mathbb{E}^{\rho}\left[\exp \left(\frac{\lambda\left(G_{\text {stat }}^{1}(\overline{\mathbf{x}})-\frac{\bar{x}}{1-\rho}\right)}{\frac{\sqrt{r+1} N^{1 / 3}}{1-\rho}}\right)\right]=\left(\frac{1}{1-\frac{\lambda}{\sqrt{\bar{x}}}} e^{-\frac{\lambda}{\sqrt{\bar{x}}}}\right)^{\bar{x}} .
$$

Now, just note that there is a constant $\delta_{0} \in(0,1)$ such that the function $\left(\frac{1}{1-\frac{y}{n}} e^{-\frac{y}{n}}\right)^{n^{2}}$ is at most $e^{C^{*} y^{2}}$ if $\frac{y}{n}<\delta_{0}$. This can be seen right away by taking a logarithm and doing a Taylor expansion.

Proof of Lemma 4.5. We will directly use Lemma 4.4. Note that $\frac{\lambda}{\sqrt{\bar{x}}}=\frac{C_{1}}{C^{*}} \frac{r^{3 / 2}}{\sqrt{r+1} N^{1 / 3}} \leq \frac{C_{1}}{C^{*}} \frac{r}{N^{1 / 3}}$, where $C_{1}$ depends only on $\rho$. Hence, by Lemma 4.4, we just require that $\frac{C_{1}}{C^{*}} \gamma_{1} \leq \delta_{0}$ which can be arranged by choosing $C^{*}$ large enough because $\delta_{0} \rightarrow 1$ as $C^{*} \rightarrow \infty$.

Recall the notation $\vec{n}$ for $(n, n)$. We now describe the coupling which implies Proposition 6.1 and Proposition 6.2 .

Proposition 7.1. For each fixed $n>0$, there exists a coupling between the two stationary representations such that for each $v \in \mathbb{Z}_{\geq 0}^{2}$ with $v \leq \vec{n}$, we have $G_{\text {stat }}^{1}(v)=G_{\text {stat }}^{2}(v)$.

Proof. The coupling proceeds by a version of the Burke property for the boundary representation of stationary LPP (Lemma 4.2 in [3]). To be precise, construct a boundary stationary model with its origin shifted to the point $(-n,-n)$; call the associated environment on $-\vec{n}+\mathbb{Z}_{\geq 0}^{2}$ as $\nu$, and let $G_{\text {stat }}^{1}(-\vec{n}, \cdot)$ denote the associated stationary passage times. From $\nu$, derive the environment $\nu^{\prime}$ on $\mathbb{Z}_{\geq 0}^{2}$ as follows:

$$
\nu_{v}^{\prime}= \begin{cases}\nu_{v}, & \text { for } v \in \mathbb{Z}_{>0}^{2} \\ G_{\text {stat }}^{1}\left(-\vec{n}, i \mathrm{e}_{1}\right)-G_{\text {stat }}^{1}\left(-\vec{n},(i-1) \mathrm{e}_{1}\right), & \text { for } v=i \mathrm{e}_{1} \\ G_{\text {stat }}^{1}\left(-\vec{n}, j \mathrm{e}_{2}\right)-G_{\text {stat }}^{1}\left(-\vec{n},(j-1) \mathrm{e}_{2}\right), & \text { for } v=j \mathrm{e}_{2} \\ 0 \text { for } v=\mathbf{0} . & \end{cases}
$$

Use $\nu^{\prime}$ to define a boundary stationary model on $\mathbb{Z}_{\geq 0}^{2}$, and use $G_{\text {stat }}^{1}(\cdot)$ for the associated stationary passage times. We now derive a point-to-line stationary model from $\nu$. Recall that we only want to define $G_{\text {stat }}^{2}(v)$ for $v \leq \vec{n}$ and we need to define the environment accordingly. Define the environment $\nu^{\prime \prime}$ to be the same 
as $\nu$ in region $\{x+y>0\} \cap\{(x, y) \leq \vec{n}\}$. Define the weight function $T$ on $\{x+y=0\} \cap\{(x, y) \leq \vec{n}\}$ as follows:

$$
T(\mathfrak{t})=G_{\text {stat }}^{1}(-\vec{n}, \mathfrak{t})-G_{\text {stat }}^{1}(-\vec{n}, \mathbf{0})
$$

Use $T$ and $\nu^{\prime \prime}$ to construct the point-to-line representation as in (2.2) and thus define $G_{\text {stat }}^{2}(v)$ for all $0 \leq v \leq n$. By an application of Lemma 4.2 in [3], the quantities $G_{\text {stat }}^{1}(\cdot)$ and $G_{\text {stat }}^{2}(\cdot)$ have the correct marginal distributions. It is easy to see that because of the coupling, we have (cf. Lemma 3.3 and Lemma 3.4 from [18])

$$
G_{\text {stat }}^{1}(v)=G_{\text {stat }}^{2}(v)=G_{\text {stat }}^{1}(-\vec{n}, v)-G_{\text {stat }}^{1}(-\vec{n}, \mathbf{0}) .
$$

for all $0 \leq v \leq \vec{n}$.

Proof of Proposition 6.1. Immediate from Proposition 7.1.

Proof of Propositon 6.2. By the almost sure uniqueness of geodesics, for either representation, the stationary geodesic to $v_{N}$ (restricted to $\mathbb{Z}_{>0}^{2}$ ) can be reconstructed just by knowing the stationary passage times for all $v$ with $\mathbf{0} \leq v \leq v_{N}$. For example, working with the point-to-line representation, if we have $G_{\text {stat }}^{2}\left(v_{N}-\mathrm{e}_{1}\right)>$ $G_{\text {stat }}^{2}\left(v_{N}-\mathrm{e}_{2}\right)$, then the stationary geodesic to $v_{N}$ is just the stationary geodesic till $v_{N}-\mathrm{e}_{1}$ concatenated with the singleton $\left\{v_{N}\right\}$, and one can proceed recursively to obtain the portion of the stationary geodesic in $\mathbb{Z}_{>0}^{2}$. By Proposition 7.1, there is a coupling for which $G_{\text {stat }}^{1}(v)=G_{\text {stat }}^{2}(v)$ for all $\mathbf{0} \leq v \leq v_{N}$. By the above discussion, this implies that the stationary geodesics till $v_{N}$ agree in the region $\mathbb{Z}_{>0}^{2}$ for both the representations and Proposition 6.2 follows immediately.

Manan Bhatia, Department of Mathematics, Indian Institute of Science, Bangalore, India

E-mail address: mananbhatia@iisc.ac.in 